\title{
Autologous conditioned plasma in anterior cruciate ligament reconstruction with hamstrings - short term clinical results
}

${ }^{1}$ UMF Tîrgu Mureş, Orthopaedics and Traumatology Department

${ }^{2}$ Clinic of Orthopaedics and Traumatology, Tîrgu Mureş

${ }^{3}$ County Hospital "Dr. Eugen Nicoară“", Reghin

\begin{abstract}
ABTRACT.
Evaluating the early clinical results of anterior cruciate ligament reconstruction using hamstrings autograft, with interference screw on the tibial side (biocomposite interference screw, ConMed-Linvatec) and continuous closed loop fixation on the femoral side (XO-Button, ConMed-Linvatec), with and without intra-articular injection of autologous conditioned plasma (ACP). Our study included 21 patients with chronic anterior cruciate ligament (ACL) ruptures for whom we performed ACL reconstruction with a hamstrings autograft. The mean age was 34 years (range, 25 to 42), 16 patients were men and 5 were women. In 10 cases we performed an intraarticular infiltration of ACP at the end of the surgical intervention. Final evaluation was performed at the end of the 6th postoperative month using the Lysholm scoring system, Tegner activity scale and objective assessment with the RolimeterTM 50A. The Lysholm score was excellent in all cases at 6 months postoperatively, with a mean Lysholm score of 90 for patients without ACP and 91.09 for patients that received ACP; the mean Tegner activity score was also similar pre- and postoperatively for the two groups (from 3.5 and 3.63 for the group without ACP and the group with ACP to 5.6 and 5.72 respectively). Joint laxity measurement was similar for both groups. We found no graft ruptures. We found similar results after ACL reconstruction with and without intra-articular injection of PRP, but further studies are necessary to determine the exact role of these substances in speeding up the recovery process in these cases.
\end{abstract}

Keywords: autologous conditioned plasma, anterior cruciate ligament, reconstruction, hamstrings

Gergely I

University of Pharmacy and Medicine Tg. Mures

$38 \mathrm{Gh}$. Marinescu, Tg. Mureş,

Tel: +40-265-215-551

\section{Introduction}

Given its location and function, the knee is subject to significant forces during physical activity, with approximately 15 to $30 \%$ of sports injuries located at this level. The most common ligament injuries of the knee are complete ruptures of the anterior cruciate ligament (ACL), which usually require surgical treatment followed by a long-term recovery program. If not treated properly, ACL injuries can cause significant functional deficits and may lead to early arthritic changes of the affected joint $[5,9,12]$.

With increased participation in sports activities population-wide, the incidence of anterior cruciate ligament injuries tends to increase. Also the expectations of professional athletes and recreational sports practitioners increased - most of them want to return to their pre-lesional level of activity as soon as possible after surgery.

ACL reconstruction is an efficient treatment of knee joint instability caused by a ruptured anterior cruciate ligament, and had become a relatively common procedure in recent years, with about 6570000 interventions/year (U.S.A.). An ACL deficient knee is associated with an increased rate of meniscal tears and degenerative changes, which is one of the important reasons for ACL reconstruction.

The use of hamstring autografts has recently increased in popularity, among patients as well. With the development and improvement of graft fixation methods, the objective biomechanical and functional results of using semitendinosus-gracilis autografts have matched and even exceeded those of bone- 
tendon-bone autograft, with the benefit of lower incidence of surgical co-morbidities - especially of anterior knee pain $[1,3,4,6,10]$.

The estimated cost of ACL reconstruction is about $2200 \mathrm{USD} /$ intervention, so any gesture that could optimize each stage of preparation, technique, recovery, social reintegration and return to sports, etc. is very important. One recent promising technique to accelerate the healing process is the use of autologous conditioned plasma or platelet-enriched plasma (ACP/PRP), with immediate applicability in ACL reconstruction. The effect of these substances is based on the local release of growth factors and cytokines by activated platelets, all of which are involved in tissue regeneration. Platelet rich plasma is prepared from the patient's venous blood, representing in fact a small volume of plasma with a high content of platelets. Once activated, these form a threedimensional network of fibrin, with local progressive release of a significant number of growth factors and proteins [2].

In this study we evaluated the early clinical results of anterior cruciate ligament reconstruction using semitendinosus and gracilis autografts, with interference screw fixation on the tibial side (biocomposite interference screw, Con-Med Linvatec) and closed-loop fixation (XO-Button, ConMed Linvatec) on the femoral side, with or without intra-articular injection of autologous conditioned plasma.

\section{Materials and method}

Between April 2011 and May 2012 we selected a number of 21 patients with chronic rupture of the ACL for whom we performed ligament reconstruction with the use of hamstring autografts at out clinic (Orthopaedics and Traumatology Clinic, Mures County Hospital).

Mean age was 34 years (range 25 to 42 years), with 16 male and 5 female patients. We divided the patients into two groups, as follows:
- Group I - 10 patients - for whom we injected 4 to $6 \mathrm{ml}$ of ACP at the end of the surgical intervention.

- Group II - 11 patients - the control group, patients that have not received ACP injections.

In all cases the intervention was started with an exploratory arthroscopy. During arthroscopic exploration we inspected the entire knee joint, with appropriate treatment of possible injuries associated with ACL rupture. In 15 cases we observed meniscal lesions that were solved by partial meniscectomy and modeling the meniscal stump in case of residual unstable portions. We also noticed cartilage injuries - they were graded based on the ICRS classification system - we found ICRS grades I and II chondral lesions on the femoral condyles and/ or tibial plateaus, without advanced degree lesions or "kissing lesions".

The nextstage of the intervention was harvesting the autografts through a skin incision on the anteriormedial face of the knee, placed 6-7 cm distal to the joint line and $2 \mathrm{~cm}$ medial to the tibial tuberosity, care being taken to avoid the sensory branch of the saphenous nerve. After subfascially identifying the tendons of the semitendinosus and gracilis muscles, these were prepared and harvested with a stripper. The obtained tendons were prepared by removing any reminiscences of muscle and reinforcing them with bioresorbable sutures. Prepared autografts were measured for length and diameter, and pre-tensioned.

We also modeled the remaining ACL stump intra-articulary, keeping a portion of it to maintain a certain degree of proprioception and facilitate autograft revascularization. We prepared the intercondylar notch by notchplasty in the cases that presented a risk of impingement (the so-called "A-shaped" notch).

The femoral and tibial tunnels were prepared separately, with the femoral tunnel made with the knee flexed at 1200 . Tunnel diameter was equal to that measured for the graft. Based on the measurement of the femoral tunnel, we chose an appropriate sized femoral implant (XO-Button), after which the implant and neoligament were retrogradely ascended through the tibial and femoral tunnels, with femoral fixation of the button on the external femoral cortex.

On the tibial side fixation was achieved by bioresorbable osteoinductive interference screws (Matryx, Con-Med Linvatec) - the size of the screw 
was chosen based on the diameter of the autograft and bone quality (generally we chose a screw $1 \mathrm{~mm}$ larger than the diameter of the autograft). The intervention was completed by intra-articular introduction of a drain and suture.

For the patients in group I we prepared 4 to 6 $\mathrm{ml}$ of autologous conditioned plasma by harvesting $15 \mathrm{ml}$ of venous blood and centrifugation at $1500 \mathrm{rpm}$ for 5 minutes (Arthrex ACP).

At 24 hours postoperatively the drain was removed and patient mobilization was started. Usually patients were discharged at 24 hours postoperatively. All patients underwent a postoperative rehabilitation protocol, which prohibited full weight-bearing on the operated limb in the first two weeks postoperatively.

Regarding the evaluation of patients, we calculated the Tegner activity score and Lysholm functional score pre- and post-operatively (at 4 weeks, 3 months and 6 months) and we measured joint laxity using a RolimeterTM 50A, preoperative and at the mentioned postoperative intervals.

\section{Results}

There were no immediate or late complications in any of the patients included in the study. All patients had full extension of the knee at 6 months after surgery, with a minimal flexion of 1300 . There were no cases of graft rupture.

Mean Lysholm score showed similar results in the two groups of patients, with a gradual increase and excellent results at 6 months postoperatively for both groups. Thus, for group I we calculated average preoperative values of 64.2 and postoperative values of 72.5 at 4 weeks, 81.6 at 3 months and 90 at 6 months. For patients in group II, the mean Lysholm scores were 66.36 preoperatively, 76.72 at 4 weeks, 84.27 at 3 months and 91.09 at 6 months postoperatively (Figure 1).

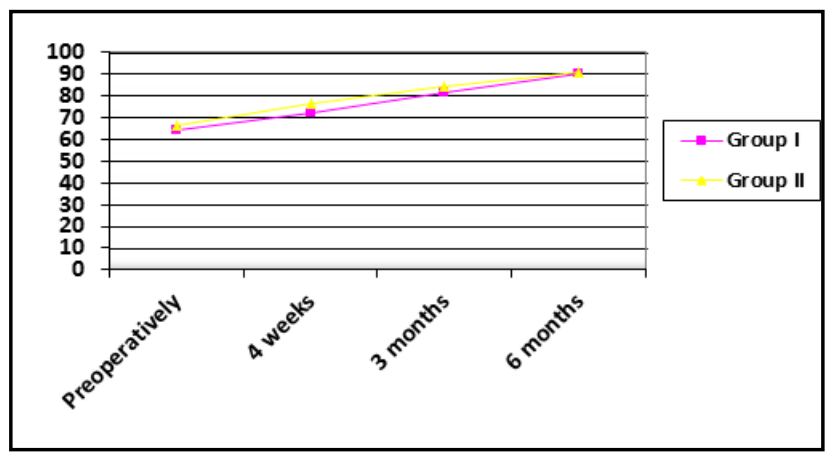

Figure 1-Mean value of the Lysholm score preoperatively and at the defined postoperative follow-up intervals

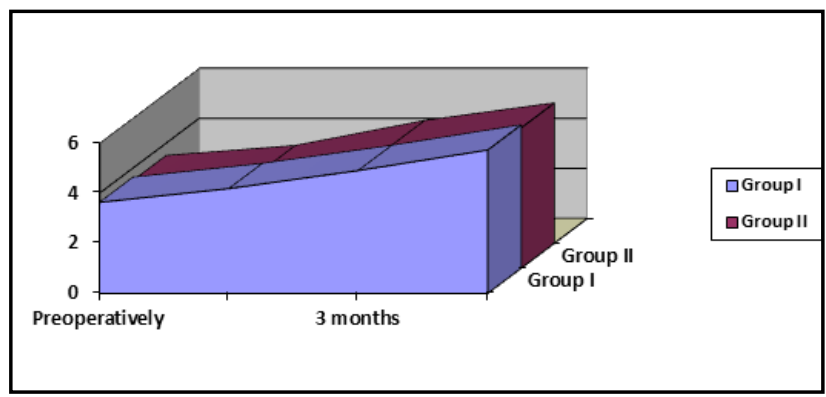

Figure 2 - Mean values of the Tegner scale preoperatively and at the postoperative follow-ups

Pre-operatively, the mean Tegner activity score was 3.63 for group I and 3.5 for group II, that increase to postoperative mean values of 4.18 and 3.9 at 4 weeks, 4.9 and 4.9 at 3 months, and 5.72 and 5.6 at 6 months postoperatively for the two groups of patients (Figure 2).

Objective evaluation using the RolimeterTM $50 \mathrm{~A}$ arthrometer presented similar pre- and postoperative results in the two groups of patients: mean displacement of $7.8 \mathrm{~mm}$ preoperatively and 8.1 for group I and group II respectively, and 1.62 and $1.45 \mathrm{~mm}$ at the last follow-up. 
actual function of intra-articular ACP injections in speeding up tissue regeneration.

The progresses obtained in molecular and cellular biology increasingly influences the orthopedic clinical practice. An eloquent example is the use of autologous conditioned plasma, a treatment modality that promises to improve the management of orthopedic disorders by inhibiting catabolic cytokines and local release of anabolic growth factors [7,14]. Autologous conditioned plasma was studied in the treatment of several diseases, including intra-articular knee injuries, and ligament injuries in particular.

Orrego and all. [8] observed a more rapid maturation of the neoligament on MRI imaging in a follow-up period of 6 months postoperatively with the use of ACP, but without affecting the bone tunnel enlargement process. In contrast Silva and all. [13] did not find a significant difference from the control group in terms of the MRI appearance of the neoligament at 3 months postoperatively. Radice and all. [11] followed a number of 50 patients between 3 and 12 months postoperatively, noting a $48 \%$ reduction of the time needed for a homogeneous signal of the graft to appear.

\section{Conclusions}

Based on our results, we believe that the use of autologous conditioned plasma in ACL reconstruction has certain advantages, but it failed to produce significantly superior results to those of the standard ligament reconstruction.

Given the fact that ACP is relatively easy to obtain and considering the data published in the literature, we believe that its use could be recommended in some situations, especially in patients with significant physical activity and athletes, for whom it is desirable to obtain a rapid recovery with early resumption of activity. However, longerterm follow-up studies are needed to determine the

\section{Acknowledgment}

This paper is partially supported by the Sectorial Operational Program Human Resources Development, financed from the European Social Fund and by the Romanian Government under the contract number POSDRU/89/1.5/S/ 60782.

\section{References}

1. Aglietti P., Giron F., Buzzi R., Biddau F. \& Sasso F. (2004). Anterior cruciate ligament reconstruction: bone-patellar tendon-bone compared with double semitendinosus and gracilis tendon grafts. A prospective, randomized clinical trial. The Journal of bone and joint surgery. American volume. 86-A(10), 21432155.

2. Anitua E., Andia I., Ardanza B., Nurden P. \& Nurden A. T. (2004). Autologous platelets as a source of proteins for healing and tissue regeneration. Thrombosis and haemostasis. 91(1), 4-15.

3. Dopirak R.M., Adamany D.C. \& Steensen R.N. (2004). A comparison of autogenous patellar tendon and hamstring tendon grafts for anterior cruciate ligament reconstruction. Orthopedics. 27(8), 837-842; quiz 843-834.

4. Ejerhed L., Kartus J., Sernert N., Kohler K. \& Karlsson J. (2003). Patellar tendon or semitendinosus tendon autografts for anterior cruciate ligament reconstruction? A prospective 
randomized study with a two-year follow-up. The American journal of sports medicine. 31(1), 19-25.

5. Fairclough J.A. (2001). BASK Instructional Lecture 2: Long-term effects of anterior cruciate injury. The Knee. 8(1), 73-74.

6. Goldblatt J.P, Fitzsimmons S.E., Balk E. \& Richmond J.C. (2005). Reconstruction of the anterior cruciate ligament: meta-analysis of patellar tendon versus hamstring tendon autograft. Arthroscopy: the journal of arthroscopic \& related surgery: official publication of the Arthroscopy Association of North America and the International Arthroscopy Association. 21(7), 791-803.

7. Nin J.R., Gasque G.M., Azcarate A.V., Beola J.D. \& Gonzalez MH. (2009). Has platelet-rich plasma any role in anterior cruciate ligament allograft healing? Arthroscopy: the journal of arthroscopic \& related surgery: official publication of the Arthroscopy Association of North America and the International Arthroscopy Association. 25(11), 1206-1213.

8. Orrego M., Larrain C. \& Rosales J. et al. (2008). Effects of platelet concentrate and a bone plug on the healing of hamstring tendons in a bone tunnel. Arthroscopy: the journal of arthroscopic \& related surgery: official publication of the Arthroscopy Association of North America and the International Arthroscopy Association. 24(12), 1373-1380.

9. Parkkari J., Pasanen K., Mattila V.M., Kannus P. \& Rimpela A. (2008). The risk for a cruciate ligament injury of the knee in adolescents and young adults: a population-based cohort study of 46500 people with a 9 year follow-up. British journal of sports medicine. 42(6), 422-426.
10. Poolman R.W., Farrokhyar F. \& Bhandari M. (2007). Hamstring tendon autograft better than bone patellar-tendon bone autograft in ACL reconstruction: a cumulative meta-analysis and clinically relevant sensitivity analysis applied to a previously published analysis. Acta orthopaedica. 78(3), 350-354.

11. Radice F., Yanez R., Gutierrez V., Rosales J., Pinedo M. \& Coda S. (2010). Comparison of magnetic resonance imaging findings in anterior cruciate ligament grafts with and without autologous platelet-derived growth factors. Arthroscopy: the journal of arthroscopic \& related surgery: official publication of the Arthroscopy Association of North America and the International Arthroscopy Association. 26(1), 50-57.

12. Segawa H., Omori G. \& Koga Y. (2001). Longterm results of non-operative treatment of anterior cruciate ligament injury. The Knee. 8(1), 5-11.

13. Silva A. \& Sampaio R. (2009). Anatomic ACL reconstruction: does the platelet-rich plasma accelerate tendon healing? Knee surgery, sports traumatology, arthroscopy: official journal of the ESSKA. 17(6), 676-682.

14. Vogrin M., Rupreht M. \& Dinevski D. et al. (2010). Effects of a platelet gel on early graft revascularization after anterior cruciate ligament reconstruction: a prospective, randomized, double-blind, clinical trial. European surgical research. Europaische chirurgische Forschung. Recherches chirurgicales europeennes. 45(2), 77-85. 